\title{
CALCULATING RUIN PROBABILITIES VIA PRODUCT INTEGRATION
}

\author{
COLIN M. RAMSAY ${ }^{1}$ \\ Actuarial Science Program \\ University of Nebraska-Lincoln \\ Lincoln NE 68588-0426, USA \\ Miguel A. USABEL ${ }^{2}$ \\ Dto. Economia Financiera y Actuarial \\ UCM Campus de Somosaguas \\ Ftad. CC Economicas \\ Madrid, Spain
}

\begin{abstract}
${ }^{3}$
When claims in the compound Poisson risk model are from a heavy-tailed distribution (such as the Pareto or the lognormal), traditional techniques used to compute the probability of ultimate ruin converge slowly to desired probabilities. Thus, faster and more accurate methods are needed. Product integration can be used in such situations to yield fast and accurate estimates of ruin probabilities because it uses quadrature weights that are suited to the underlying distribution. Tables of ruin probabilities for the Pareto and lognormal distributions are provided.
\end{abstract}

\section{KEYWORDS}

Integral equation, convergence, heavy-tailed distributions.

\section{INTRODUCTION}

Let us consider the classical compound Poisson risk model with nonnegative claims. Specifically, let $u$ be the initial risk reserv, $F($.$) be the cumulative distribution function$ of the nonnegative claim size random variable, $p_{1}$ be the expected claim size, $1+\theta$ be the loading factor applied to the net premium rate, and $\psi(u)$ be the infinite time probability of ruin for an initial risk reserve of $u$.

Gerber (1979, p. 115, equation (3.7)) has shown that $\psi(u)$ satisfies the following Volterra integral equation of the second kind:

\footnotetext{
'Internet address: cramsay @unlinfo.unl.edu

${ }^{2}$ Internet address: econ109@sis.ucm.es

${ }^{3}$ This research was done at the University of Nebraska-Lincoln while Dr. Usabel was visiting his postdoctoral researcher. The authors gratefully acknowledge the financial support from Universidad Complutense de Madrid (Ayudas Postdoctorales en el Extranjero) and from the E.J. Faulkner Chair at the University of Nebraska-Lincoln. 


$$
\psi(u)=\frac{1}{1+\theta}\left[A(u)+\int_{0}^{u} K(u, t) \psi(t) d t\right], \quad u \geq 0
$$

where

$$
\begin{gathered}
a(u)=\int_{u}^{\infty} \frac{1-F(t)}{p_{1}} d t, \quad u \geq 0 \\
K(u, t)=\frac{1-F(u-t)}{P_{1}}, \quad 0 \leq t \leq u .
\end{gathered}
$$

A classic problem (of interest mainly to academic actuaries) is the numerical evaluation $\psi(u)$. Numerous authors have studied this problem; see, for example, recent texts by Grandell (1991) and Panjer and Willmot (1992, Chapter 11) and references therein. In general, no explicit closed form solution to equation (1) exists except in the case where claims are mixtures of exponential distributions; see Bowers et al. (1986, Chapter 12.6).

There are, however, several broad approaches to the evaluation $\psi(u)$. The older approaches are ad hoc: focusing inverting the Laplace transform, or on matching the first few moments of the claim size distribution or on the Cramer-Lundberg approximation; see Ramsay (1992a) for a comparison of some of these methods.

Since the early 1980s, the shift has been to approaches based on discretizing some aspect of the risk process and deriving recursive expressions for $\psi(u)$; see, for example, Goovaerts and De Vylder (1984), Panjer (1986), Dickson (1989), Dickson and Waters (1991), Ramsay (1992b), and Dickson, Egidio dos Reis and Waters (1995). Panjer and Wang (1993) describe the conditions under which these recursions are stable.

Though these recursive approaches may be able to determine $\psi(u)$ to any desired degree of accuracy, they are not suitable for heavy-tailed distributions, such as the Pareto or lognormal distributions, for two main reasons:

1. To achieve a reasonable degree of accuracy, the interval of discretization must be at most one unit of the mean in lenght. If we standardize the unit of currency such that $p_{1}=1$, then to obtain $\psi(10)$ we must recursively estimate every intermediate unit point $\psi(u)$ for $k=0,1,2, \ldots, 9,10$. This may be acceptabel if we need only small values of $u$; however, for large values of $u$, say $u=500$ units, this method can be slow. For the Pareto, $\psi(500)$ is not insignificant.

2. The quadrature rules inherent in the recursive schemes are usually of low order. This further reduces its accuracy and its rate of convergence. To improve accuracy, the intervals of discretization are made even smaller. This substantially increases the number of intermediate calculations required, making the process of finding $\psi(u)$ slower.

The objective of this paper is to present a method of evaluating $\psi(u)$ using socalled product integration. We show that this method can be fast and accurate when dealing with heavy-tailed distributions. 


\section{PRODUCT INTEGRATION}

Consider the numerical solution of the Volterra integral equation

$$
x(s)=y(s)+\int_{a}^{s} k(s, t) x(t) d t, \quad a \leq s \leq b
$$

where $k(.,$.$) is the kernel (and is known) and x($.$) is the unknown function to be deter-$ mined. Assume $k(.,$.$) or one of its low-order derivatives is badly behaved in one of its$ arguments. (For example, $k(.,$.$) may be singular or nearly singular). In such a situation,$ the Newton-Cotes integration (e.g., trapezoid rule, Simpson's rule, etc.) may produce inaccurate results or suffer a reduced rate of convergence.

Delves and Mohamed (1985) and Linze (1985) recommend the use of product integration' to take account of the fact that $k(.,$.$) may be badly behaved. Our development$ of the product integration quadrature rule follows the exposition and notation of Delves and Mohamed (Chapters 4.4 and 5.5). For a more detailed description of the product integration technique, see Linz (1985, Chapter 8).

First we factorize $k(s, t)$ as

$$
k(s, t)=p(s, t) \bar{k}(s, t)
$$

where $\bar{k}(.,$.$) is smooth and well-behaved and can be accurately approximated by a$ suitable Lagrangian interpolating polynomial, and $p(s, t)$ is badly behaved. Next we decompose the interval $[a, b]$ into $n$ subintervals $\left\{h_{i}\right\}$ where

$$
h_{i}=s_{i+1}-s_{i}, \quad i=0,1, \ldots, n-1
$$

and

$$
a=s_{0}<s_{1}<\ldots<s_{n}=b .
$$

Product integration proceeds by approximating the integral in equation (4) for $s=s_{i}$, $i=1,2, \ldots, n$, using a quadrature rule of the form

$$
\int_{a}^{s_{i}} p\left(s_{i}, t\right) \bar{k}\left(s_{i}, t\right) x(t) d t \approx \sum_{j=0}^{i} w_{i j} \bar{k}\left(s_{i}, t\right) x\left(t_{j}\right)
$$

where $t_{i}=s_{i}$ for $i=0,1,2, \ldots, n$. The weights are determined by insuring that the rule of equation (5) is exact when $\bar{k}(s, t) x(t)$ is a polynomial in $t$ of degree $\leq d$. Product integration is only applicable if the following $(d+1)$ moments $\mu_{i j}$ exist and can be calculated for each $i$, where

$$
\mu_{i j}=\int_{a}^{s_{i}} t^{j} p\left(s_{i}, t\right) d t, \quad j=0,1, \ldots, d .
$$

In this paper we assume $\bar{k}\left(s_{i}, t\right) x(t)$ is linear $(d=1)$ in $t$, i.e.,

$$
\bar{k}\left(s_{i}, t\right) x(t) \approx \frac{\left(t_{j+1}-t\right)}{h_{j}} \bar{k}\left(s_{i}, t\right) x\left(t_{j}\right)+\frac{\left(t-t_{j}\right)}{h_{j}} \bar{k}\left(s_{i}, t_{j+1}\right) x\left(t_{j+1}\right) .
$$

\footnotetext{
'Linz (1985, Chapter 8, p. 141) attributes the origin of the product integration technique to Young
} (1954). 
It follows that

$$
\begin{aligned}
\int_{a}^{s_{i}} p\left(s_{i}, t\right) \bar{k}\left(s_{i}, t\right) x(t) d t \approx & \sum_{j=0}^{i-1} \int_{t_{j}}^{t_{j+1}} p\left(s_{i}, t\right)\left[\frac{\left(t_{j+1}-t\right)}{h_{j}} \bar{k}\left(s_{i}, t_{j}\right) x\left(t_{j}\right)\right. \\
& \left.+\frac{\left(t-t_{j}\right)}{h_{j}} \bar{k}\left(s_{i}, t_{j+1}\right) x\left(t_{j+1}\right)\right] \\
= & \sum_{j=0}^{i} w_{i j} \bar{k}\left(s_{i}, t_{j}\right) x\left(t_{j}\right)
\end{aligned}
$$

where

$$
\begin{aligned}
w_{i 0}= & \int_{t_{0}}^{t_{1}} p\left(s_{i}, t\right) \frac{\left(t_{1}-t\right)}{h_{0}} d t \quad \text { for } j=0 \\
w_{i j}= & \int_{t_{j}}^{t_{j+1}} p\left(s_{i}, t\right) \frac{\left(t_{j+1}-t\right)}{h_{j}} d t \\
& +\int_{t_{j-1}}^{t_{j}} p\left(s_{i}, t\right) \frac{\left(t-t_{j-1}\right)}{h_{j-1}} d t \quad \text { for } j=1,2, \ldots, i-1 \\
w_{i j}= & \int_{t_{i-1}}^{t_{i}} p\left(s_{i}, t\right) \frac{\left(t-t_{i-1}\right)}{h_{i-1}} d t \quad \text { for } j=i
\end{aligned}
$$

To facilitate easy computation of the weights, we introduce two new variables:

$$
\begin{gathered}
v_{i j}=\int_{t_{j}}^{t_{j+1}}\left(t_{j+1}-t\right) p\left(s_{i}, t\right) d t \\
c_{i j}=\int_{i_{j}}^{t_{j+1}} p\left(s_{i}, t\right) d t .
\end{gathered}
$$

As $t-t_{j}=\left(t_{j+1}-t_{j}\right)-\left(t_{j+1}-t\right)$, then

$$
\begin{aligned}
& w_{i 0}=\frac{v_{i 0}}{h_{0}} \\
& w_{i j}=\frac{v_{i j}}{h_{j}}+c_{i j}-\frac{v_{i, j-1}}{h_{j-1}} \text { for } j=1,2, \ldots, i-1 \\
& w_{i i}=c_{i, i-1}-\frac{v_{i, i-1}}{h_{i-1}} .
\end{aligned}
$$

Thus, the approximate solution to equation (4) is determined recursively using

$$
\hat{x}_{n}\left(s_{i}\right)=y\left(s_{i}\right)+\sum_{j=0}^{i} w_{i j} \bar{k}\left(s_{i}, t_{j}\right) \hat{x}_{n}\left(t_{j}\right)
$$

for $i=1,2, \ldots, n$, with

$$
\hat{x}_{n}\left(s_{0}\right)=y(a) .
$$

The resulting estimate of $x(s)$ is $\hat{x}_{n}\left(s_{n}\right)$. 


\section{ACCELERATING THE CONVERGENCE}

We can improve the accuracy of our estimate $\hat{x}_{n}(s)$ by dividing the interval $[a, s]$ into smaller subintervals. Following the arguments of Ramsay (1992), Richardson's extrapolation technique can be used to accelerate the convergence of $\hat{x}_{n}(s)$ to $x(s)$ as $n \rightarrow \infty$. To this end, let us divide the interval $[a, s]$ into $n_{j}$ intervals of equal length, where

$$
n_{j}=\gamma \times 2^{j} \quad j=0,1,2, \ldots
$$

and $\gamma$ is a positive integer. For given $j$ and $[a, s]$, we have

$$
\begin{aligned}
s_{n_{j}} & =s \\
h & =(s-a) / n_{j} \quad \text { for } i=0,1,2, \ldots, n_{j}-1 \\
s_{i}=t_{i} & =a+i h \text { for } i=0,1,2, \ldots, n_{j}-1
\end{aligned}
$$

The Richardson extrapolation technique generates a lower diagonal matrix of approximations:

$$
T_{r}^{j}=T_{r-1}^{j}+\frac{T_{r-1}^{j}-T_{r-1}^{j-1}}{2^{r}-1}
$$

for $r=1,2, \ldots, j$ and $j=1,2, \ldots$ with $T_{0}^{j}=\hat{x}_{n_{j}}(s)$. The final estimate of $x(s)$ is:

$$
\hat{x}(s)=T_{J}^{j} .
$$

\section{THE MAIN RESULTS}

Product integration is used to compute ruin probabilities for the Pareto and lognormal distributions. Without loss of generality, set $p_{1}=1$ for each distribution. Tables 1 and 2 show the final estimated values of the ruin probabilities after the Richardson extrapolation technique has been applied.

\subsection{The Pareto Distribution}

Consider the Pareto distribution defined on $(0, \infty)$ with unit mean, i.e.,

$$
F(t)=1-\left(\frac{\alpha}{\alpha+t}\right)^{\alpha+1} \quad \alpha>0 \text { and } t>0 .
$$

Equations (2) and (3) imply

$$
\begin{aligned}
A(u) & =\left(\frac{\alpha}{\alpha+u}\right)^{\alpha} \\
K(u, t) & =\left(\frac{\alpha}{\alpha+u-t}\right)^{\alpha+1}
\end{aligned}
$$

Even though $K(u, t)$ and all of its derivatives are smooth and wellbehaved, they converge slowly as $u \rightarrow \infty$. As all of the moments $\mu_{i j}$ exist for any finite $s$, product integration can be used. 
Next set

$$
\begin{aligned}
& p(s, t)=K(s, t) \\
& \bar{k}(s, t)= \begin{cases}1 & \text { if } 0 \leq t \leq s ; \\
0 & \text { otherwise. }\end{cases}
\end{aligned}
$$

To determine the product integration weights, we need $v_{i j}$ and $c_{i j}$ from equations (6) and (7).

$$
v_{i j}=d_{i j}+\left(\alpha+s_{i}-t_{j+1}\right) c_{i j}
$$

where

$$
\begin{aligned}
& d_{i j}=\left\{\begin{array}{lc}
\ln \left(1+s_{i}-t_{j}\right)-\ln \left(1+s_{i}-t_{j+1}\right) & \text { if } \alpha \neq 1 ; \\
\frac{\alpha^{2}}{\alpha-1}\left[\left(\frac{\alpha}{\alpha+s_{i}-t_{j}}\right)^{\alpha-1}-\left(\frac{\alpha}{\alpha+s_{i}-t_{j+1}}\right)^{\alpha-1}\right] & \text { if } \alpha \neq 1 .
\end{array}\right. \\
& c_{i j}=\left(\frac{\alpha}{\alpha+s_{i}-t_{j}}\right)^{\alpha}-\left(\frac{\alpha}{\alpha+s_{i}-t_{j+1}}\right)^{\alpha} .
\end{aligned}
$$

Tabel 1 shows the ruin probabilities for the Pareto distribution with $\alpha=1$ and several values of $\theta$. From equation (13), we use $\gamma=20$ and $j=0,1,2,3$ and 4 . (Thus, $n_{4}=$ 320.)

TABLE 1

Ruin Probabilities: Pareto Distribution $(\alpha=1)$

\begin{tabular}{cccccc}
\hline \hline $\boldsymbol{\Psi}(\mathbf{u})$ for Various & Values of $\theta$ & & & & \\
$\boldsymbol{u}$ & $\theta=\mathbf{0 . 1 0}$ & $\theta=\mathbf{0 . 2 5}$ & $\theta=\mathbf{0 . 5 0}$ & $\theta=\mathbf{0 . 7 5}$ & $\theta=\mathbf{I . 0 0}$ \\
\hline 10 & 0.627128 & 0.372677 & 0.206646 & 0.138242 & 0.102523 \\
20 & 0.498142 & 0.245260 & 0.119274 & 0.075908 & 0.055049 \\
30 & 0.411437 & 0.178338 & 0.081426 & 0.051056 & 0.036887 \\
40 & 0.347893 & 0.137559 & 0.060856 & 0.038038 & 0.027509 \\
50 & 0.299155 & 0.110519 & 0.048164 & 0.030142 & 0.021847 \\
60 & 0.260646 & 0.091524 & 0.039650 & 0.024884 & 0.018080 \\
70 & 0.229551 & 0.077594 & 0.033588 & 0.021150 & 0.015402 \\
80 & 0204018 & 0.067029 & 0.029075 & 0.018369 & 0.013404 \\
90 & 0.182761 & 0.058794 & 0.025596 & 0.016222 & 0.011859 \\
100 & 0.164860 & 0.052227 & 0.022839 & 0.014517 & 0.010630 \\
200 & 0.076323 & 0.023800 & 0.010860 & 0.007028 & 0.005194 \\
300 & 0.046612 & 0.015154 & 0.007083 & 0.004621 & 0.003429 \\
400 & 0.032827 & 0.011071 & 0.005247 & 0.003438 & 0.002557 \\
500 & 0.025123 & 0.008708 & 0.004165 & 0.002737 & 0.002038 \\
600 & 0.020273 & 0.007170 & 0.003451 & 0.002273 & 0.001694 \\
700 & 0.016962 & 0.006092 & 0.002946 & 0.001943 & 0.001449 \\
800 & 0.014566 & 0.005294 & 0.002569 & 0.001696 & 0.001266 \\
900 & 0.012756 & 0.004681 & 0.002278 & 0.001505 & 0.001124 \\
1000 & 0.011341 & 0.004194 & 0.002046 & 0.001353 & 0.001011 \\
\hline
\end{tabular}




\subsection{Lognormal Distibution}

In this case things will be more complicated because of the presence of the normal cumulative distribution function. Again we assume that $p_{1}=1$. This implies

$$
\begin{aligned}
A(u) & =\int_{u}^{\infty} 1-F(t) d t, \quad u \geq 0 \\
K(u, t) & =1-\Phi\left(\frac{\ln (u-t)-\mu}{\sigma}\right), \quad 0 \leq t \leq u \\
\mu & =e^{-\sigma^{2} / 2 \quad\left(\text { as } p_{1}=1\right)}
\end{aligned}
$$

where $\mu$ and $\sigma$ are the parameters of the lognormal and

$$
\Phi(u)=\int_{-\infty}^{u} \frac{e^{-t^{2} / 2}}{\sqrt{2 \pi}} d t .
$$

A source of difficulty is in the computation of $v_{i j}$ adn $c_{i j}$, i.e.,

$$
\begin{aligned}
& v_{i j}=\int_{t_{j}}^{t_{j+1}}\left(t_{j+1}-t\right)\left(1-\Phi\left(\frac{\ln \left(s_{i}-t\right)-\mu}{\sigma}\right)\right) d t \\
& c_{i j}=\int_{t_{j}}^{t_{j+1}}\left(1-\Phi\left(\frac{\ln \left(s_{i}-t\right)-\mu}{\sigma}\right)\right) d t .
\end{aligned}
$$

As the function $\Phi($.$) is known only approximately, these integrals must be computed$ numerically; see for example Abramowitz and Stegun (1964, Chapter 26) for several approximations. The approximation used in this paper is:

$$
\Phi(u)=1-\frac{e^{-u^{2} / 2}}{\sqrt{2 \pi}}\left(\sum_{k=1}^{5} b_{k} t^{k}\right)+\varepsilon(u)
$$

where $|\varepsilon(u)|<7.5 \times 10^{-8}$, and

$$
\begin{aligned}
t=1 /(1+p u) & p=0.2316419 \\
b_{1}=0.319381530 & b_{4}=-1.821255978 \\
b_{2}=-0.356563782 & b_{5}=1.330274429 \\
b_{3}=1.781477937 &
\end{aligned}
$$

Gaussian integration rules many be used to evalutate the integrals.

Table 2 shows the ruin probabilities for the lognormal distribution with $\sigma=1.80$ and several values of $\theta$. From equation (13), we use $\gamma=10$ and $j=0,1,2,3$ and 4. (Thus, $n_{4}=160$. These values are very close to those of Thorin and Wikstad (1977), where appropriate. 
TABLE 2

Ruin Probabilities: Lognormal Distribution $(\sigma=1.80)$

\begin{tabular}{cccccc}
\hline \hline $\boldsymbol{\Psi}(\mathbf{u})$ for Various Values of $\boldsymbol{u}$ and $\theta$ & & & & \\
$\boldsymbol{u}$ & $\boldsymbol{\theta} \mathbf{0 . 1 0}$ & $\theta=\mathbf{0 . 2 5}$ & $\boldsymbol{\theta} \mathbf{0 . 5 0}$ & $\boldsymbol{\theta = 0 . 7 5}$ & $\boldsymbol{\theta}=\mathbf{1 . 0 0}$ \\
\hline 10 & 0,739768 & 0,518832 & 0,336874 & 0,245749 & 0,192154 \\
20 & 0,656692 & 0,410781 & 0,240187 & 0,165669 & 0,125229 \\
30 & 0,593553 & 0,339538 & 0,184539 & 0,122940 & 0,091161 \\
40 & 0,541731 & 0,287396 & 0,147713 & 0,096077 & 0,070371 \\
50 & 0,497634 & 0,247190 & 0,121512 & 0,077676 & 0,056424 \\
60 & 0,459303 & 0,215164 & 0,101989 & 0,064361 & 0,046484 \\
70 & 0,425505 & 0,189068 & 0,086956 & 0,054343 & 0,039091 \\
80 & 0,395396 & 0,167437 & 0,075086 & 0,046580 & 0,033413 \\
90 & 0,368362 & 0,149265 & 0,065528 & 0,040423 & 0,028940 \\
100 & 0,343939 & 0,133830 & 0,057704 & 0,035446 & 0,025344 \\
200 & 0,188093 & 0,055553 & 0,022128 & 0,013482 & 0,009651 \\
300 & 0,113139 & 0,029147 & 0,011567 & 0,007112 & 0,005124 \\
400 & 0,072445 & 0,017524 & 0,007067 & 0,004390 & 0,003180 \\
500 & 0,048684 & 0,011534 & 0,004747 & 0,002974 & 0,002164 \\
600 & 0,034048 & 0,008096 & 0,003397 & 0,002143 & 0,001565 \\
700 & 0,024637 & 0,005960 & 0,002544 & 0,001614 & 0,001182 \\
800 & 0,018360 & 0,004551 & 0,001971 & 0,001257 & 0,000922 \\
900 & 0,014040 & 0,003577 & 0,001569 & 0,001004 & 0,000738 \\
1000 & 0,010981 & 0,002878 & 0,001276 & 0,000819 & 0,000603 \\
\hline \hline
\end{tabular}

\section{CONCLUDING COMMENTS}

The important strength of the product integration technique in solving equation (1) is that it converges significantly faster and is more accurate than the Goovaerts and de Vylder (1984) technique, or the improved version proposed by Ramsay (1992b). This is acheived by using a quadrature rule that exploits some of the features of the kernel, thus requiring a reduced amount of recursions. Even though the weights $w_{i j}$ (and hence $c_{i j}$ and $v_{i j}$ ) have to be computed directly from the kernel, these extra computations are fast and easy to perform.

Because product integration converges relatively rapidly, it does not require the use of small intervals, thus reducing the possibilitiy of subtracting nearly equal numbers (and hence rounding errors). In addition, it requires a small fraction of the computations required by the Goovaerts-De Vylder-Ramsay approach to obtain the same degree of accuracy. This should not be surprising because product integration uses much more information from the integrand than do the common Newton-Cotes quadrature formulae.

A further area of research is the determination of the error bounds of the solutions generated via the product integration technique. Linz (1985, Chapter 8, p. 131) shows that the error bounds and orders of convergence for product integration follow the standard results of approximation theory. Thus, product integration based on the trapezoidal rule is of order $O\left(h^{2}\right)$.

Additionally, one may be able to use the Goovaerts-De Vylder-Ramsay approach and combine it with product integration to produce a faster scheme with explicit error bounds. 


\section{REFERENCES}

Abramowitz, M. and Stegun, I.A. (1964). Handbook of Mathematical Functions. New York, N.Y.: Dover Publications.

Bowers, N.L., Gerber, H.U., Hickman, J.C., Jones, D.A. and NesbitT, C.J. (1986). Actuarial Mathematics. Ithasca, Ill.: Society of Actuaries.

Delves, L.M. and Mohamed, J.L. (1989) Computational Methods for Integral Equations. Cambridge, England: Cambridge University Press.

DickSON, D.C.M. (1989). "Recursive Calculation of the Probability and Severity of Ruin." Insurance: Mathematics and Economics, 8, pp. 145-148.

Dickson, D.C.M., Egidio dos Reis, A.D. and Waters, H.R. (1995). "Some Stable Algorithms in Ruin Theory and Their Application." Astin Bulletin 25, pp. 153-175.

Dickson, D.C.M. and WATERS, H.R. (1991) "Recursive Calculation of Survival Probabilities." Astin Bulle$\operatorname{tin} 21$, pp. 199-221.

Gerber, H.U. (1979) An Introduction to Mathematical Risk Theory. Huebner Foundation Monograph 8. Philadelphia, Pa.: University of Pennsylvania. (Distributed by Irwin, Homewood, IL.)

GoovaerTs, M. and DE VyLder, F. (1984) "A Stable Recursive Algorithm for Evaluation of Ultimate Ruin Probabilities." Astin Bulletin, 14, pp. 53-59.

GRandELl,J. (1990). Aspects of Risk Theory. Springer Verlag, New York.

Linz, P. (1985). Analytical and Numerical Methods for Volterra Equations. Philadelphia, Pa: SIAM Studies in Applied Mathematics.

PANJER, H.H. (1986) "Direct Calculation of Ruin Probabilities." Journal of Risk and Insurance, 53, pp. 521529.

PANJer, H.H. and WANG, S (1993) "On the Stability of Recursive Formulas." ASTIN Bulletin , 23, pp. 227258.

RaMSAY, C.M. (1992a) "A Practical Algorithm for Approximating the Probability of Ruin." Transactions of the Society of Actuaries, XLIV, 443-459.

RaMSAY, C.M. (1992b) "Improving Goovaerts' and de Vylder's Stable Recursive Algorithm." Astin Bulletin, 22, pp. 51-59.

Thorin, O. and WIKSTAD, N. (1977) "Calculation of Ruin Probabilities When the Claim Distribution is Lognormal.” Astin Bulletin, 9, pp. 231-246.

Young, A. (1954) "Approximate Product Integration." Proc. Royal Soc. London, Ser. A, 224, pp. 561-573. 